\title{
Télescope
}

Revue d'analyse comparée en administration publique

\section{La transparence gouvernementale et le cybergouvernement : les enjeux démocratiques selon une perspective publique}

\author{
Teresa M. Harrison, Santiago Guerrero, G. Brian Burke, Meghan Cook, Anthony \\ Cresswell, Natalie Helbig, Jana Hrdinová et Theresa Pardo
}

Volume 18, numéro 1-2, printemps-été 2012

Les administrations publiques à l'ère du numérique

URI : https://id.erudit.org/iderudit/1009252ar

DOI : https://doi.org/10.7202/1009252ar

Aller au sommaire du numéro

Éditeur(s)

L’Observatoire de l'administration publique

ISSN

1203-3294 (imprimé)

1929-3348 (numérique)

Découvrir la revue

Citer cet article

Harrison, T. M., Guerrero, S., Burke, G. B., Cook, M., Cresswell, A., Helbig, N., Hrdinová, J. \& Pardo, T. (2012). La transparence gouvernementale et le cybergouvernement : les enjeux démocratiques selon une perspective publique. Télescope, 18(1-2), 1-20. https://doi.org/10.7202/1009252ar

\section{Résumé de l'article}

Dans cet article, nous abordons la notion de transparence gouvernementale et sa portée générale sur l'avenir de l'administration publique dans un contexte de cybergouvernement. Nous soutenons que l'Open Government Initiative de l'actuelle administration américaine estompe les différences conventionnelles entre cyberdémocratie et cybergouvernement par l'intégration, au moyen des technologies, de pratiques démocratiques traditionnelles dans les organismes administratifs. Nous examinons comment fonctionnent les pratiques démocratiques axées sur la transparence, la participation et la collaboration dans les organismes administratifs, en supposant qu'elles contribuent à l'action administrative et au processus décisionnel, contrairement à l'approche actuelle qui semble les considérer comme la finalité de l'action administrative. Nous étudions le gouvernement transparent sous l'angle de la « valeur publique » que souhaitent produire les organismes publics, car elle leur permet de combler les besoins et les aspirations de la population par ses avantages considérables et la valeur intrinsèque associée à un meilleur gouvernement. Nous appliquons cette vision à la notion de transparence gouvernementale pour décrire la valeur dégagée par une interaction gouvernement-citoyen fondée sur la transparence, la participation et la collaboration, c'est-à-dire une interaction plus démocratique.
Tous droits réservés (C L’Observatoire de l'administration publique, 2012
Ce document est protégé par la loi sur le droit d'auteur. L’utilisation des services d'Érudit (y compris la reproduction) est assujettie à sa politique d'utilisation que vous pouvez consulter en ligne.

https://apropos.erudit.org/fr/usagers/politique-dutilisation/ 


\section{LA TRANSPARENCE \\ GOUVERNEMENTALE ET LE \\ CYBERGOUVERNEMENT : LES ENJEUX \\ DÉMOCRATIQUES SELON UNE \\ PERSPECTIVE PUBLIQUE}

Par Teresa M. Harrison ${ }^{1}$, Professeure, Center for Technology in Government, University at Albany, États-Unis • tharrison@albany.edu

Santiago Guerrero, Doctorant, Rockefeller College of Public Affairs \& Policy, University at Albany, États-Unis • sangue20@yahoo.com

G. Brian Burke, Agent principal de programme, Center for Technology in Government, University at Albany, États-Unis • gburke2@albany.edu

Meghan Cook, Gestionnaire de programme, Center for Technology in Government, University at Albany, États-Unis • mcook@ctg.albany.edu

Anthony Cresswell, Collaborateur émérite, Center for Technology in Government, University at Albany, États-Unis • acresswell@ctg.albany.edu

Natalie Helbig, Agente principale de programme, Center for Technology in Government, University at Albany, États-Unis • nhelbig@ctg.albany.edu

Jana Hrdinová, Agente de programme, Center for Technology in Government, University at Albany, États-Unis • jhrdinova@ctg.albany.edu

Et Theresa Pardo, Directrice, Center for Technology in Government, University at Albany, États-Unis • tpardo@ctg.albany.edu

Traduit de l'anglais ${ }^{2}$

RÉSUMÉ Dans cet article, nous abordons la notion de transparence gouvernementale et sa portée générale sur l'avenir de l'administration publique dans un contexte de cybergouvernement. Nous soutenons que l'Open Government Initiative de l'actuelle administration américaine estompe les différences conventionnelles entre cyberdémocratie et cybergouvernement par l'intégration, au moyen des technologies, de pratiques démocratiques traditionnelles dans les organismes administratifs. Nous examinons comment fonctionnent les pratiques démocratiques axées sur la transparence, la participation et la collaboration dans les organismes administratifs, en supposant qu'elles contribuent à l'action administrative et au processus décisionnel, contrairement à l'approche actuelle qui semble les considérer

1 Cet article s'appuie sur des travaux financés en partie par la National Science Foundation ( $n^{\circ} 0956356$ ) des États-Unis. Toutes opinions, découvertes, conclusions ou recommandations exprimées dans le présent document sont celles des auteurs et ne reflètent pas nécessairement les vues de la National Science Foundation.

2 Cet article a été reproduit avec la permission de l'Association for Cumputing Machinery (ACM). Cette traduction est un dérivé de matériel sous droit d'auteur d'ACM. ACM n'a pas préparé cette traduction et ne garantit pas que ce soit une copie exacte de l'œuvre originale. La propriété intellectuelle originale contenue dans cet ouvrage reste la propriété de I'ACM. Harrison, T. M. et al. Open Government and e-Government: Democratic Challenges from a Public Value Perspective, Proceedings of the 12th Annual International Digital Government Research Conference: Digital Government Innovation in Challenging Times, (c) 2011 ACM, Inc. http://dx.doi.org/10.1145/2037556.2037597. 
comme la finalité de l'action administrative. Nous étudions le gouvernement transparent sous l'angle de la " valeur publique " que souhaitent produire les organismes publics, car elle leur permet de combler les besoins et les aspirations de la population par ses avantages considérables et la valeur intrinsèque associée à un meilleur gouvernement. Nous appliquons cette vision à la notion de transparence gouvernementale pour décrire la valeur dégagée par une interaction gouvernement-citoyen fondée sur la transparence, la participation et la collaboration, c'est-à-dire une interaction plus démocratique.

\begin{abstract}
We consider open government (OG) within the context of e-Government and its broader implications for the future of public administration. We argue that the current US Administration's Open Government Initiative blurs traditional distinctions between e-Democracy and e-Government by incorporating historically democratic practices, now enabled by emerging technology, within administrative agencies. We consider how transparency, participation, and collaboration function as democratic practices in administrative agencies, suggesting that these processes are instrumental attributes of administrative action and decision making, rather than the objective of administrative action, as they appear to be currently treated. We propose alternatively that planning and assessing OG be addressed within a "public value" framework. The creation of public value is the goal of public organizations; through public value, public organizations meet the needs and wishes of the public with respect to substantive benefits as well as the intrinsic value of better government. We extend this view to OG by using the framework as a way to describe the value produced when interaction between government and citizens becomes more transparent, participative, and collaborative - i.e., more democratic.
\end{abstract}

Pour citer cet article : Harrison, T. M. et autres (2012). « La transparence gouvernementale et le cybergouvernement : les enjeux démocratiques selon une perspective publique », Télescope, vol. 18, n 1-2, p. 1-20.

$\mathrm{O}_{\mathrm{p}}^{\mathrm{n}}$ considère généralement que l'utilisation d'Internet et des médias sociaux par Barack Obama durant sa campagne présidentielle en 2008 a révolutionné l'art contemporain de la campagne électorale (Stirland, 2008). Ainsi, à l'issue d'une campagne qui avait misé sur la participation stratégique de bénévoles et sur l'organisation d'une variété d'échanges entre les électeurs et le candidat, la victoire était à peine proclamée que les prédictions circulaient quant à la volonté d'Obama d'en transposer les grandes lignes à l'administration quotidienne du pouvoir exécutif (Stone et Lyons, 2008). En sa qualité de premier titulaire de la "présidence Internet " (Wagner, 2008), le président désigné, appuyé par son équipe de transition, a rapidement concrétisé ces prédictions. Le 21 janvier 2009, à l'occasion de l'une de ses premières mesures administratives, le président Obama a publié une circulaire présidentielle sur la transparence et le gouvernement ouvert (Obama, 2009) qui chargeait l'Office of Management and Budget d'adopter dans les cent vingt jours une directive sur la transparence dans l'administration publique. La circulaire posait la transparence, la participation et la collaboration en principes fondamentaux d'un gouvernement ouvert.

La directive sur la transparence gouvernementale publiée le 8 décembre 2009 met au premier plan ces principes essentiels de transparence, de participation et de collaboration qui forment " la pierre angulaire du gouvernement transparent " (Orszag, 2009). Elle somme les organismes fédéraux « d'appliquer ces principes » en 
améliorant l'accessibilité aux documents et la qualité des renseignements de l'État, ainsi qu'en institutionnalisant une " culture de transparence gouvernementale " fondée sur la mobilisation de gens d' "expertise et de discernement " et l'établissement de "solides liens de collaboration avec les chercheurs, le secteur privé et la société civile " (Orszag, 2009, p. 5). En raison de leur possible capacité " d'ouvrir de nouvelles voies de communication entre le gouvernement et la population " (Orszag, 2009, p. 5), les technologies pourraient jouer un rôle central dans cette entreprise. La directive demande également aux organismes fédéraux concernés de recenser les politiques en place qui pourraient entraver le recours aux technologies dans la promotion des visées de la transparence gouvernementale et de suggérer des révisions. Les organismes qui ont embrassé la directive ont par la suite fait un usage considérable d'Internet, du Web et des nouvelles possibilités offertes par les médias sociaux dans le cadre de leurs programmes de transparence ${ }^{3}$. Dans cet esprit, l'administration Obama a posé un geste d'envergure en redéfinissant complètement l'orientation du cybergouvernement sur la scène fédérale pour au moins les trois prochaines années.

Pour mieux apprécier le caractère novateur de l'Open Government Initiative, il y a lieu de la comparer à celles des administrations précédentes. Dans les années 1990, l'administration Clinton a entrepris un examen national du rendement et des partenariats subséquents dans le cadre de l'initiative Reinventing Government. Celle-ci s'appuyait sur le recours à la technologie dans les services administratifs en vue d'améliorer les processus et sur la création de sites Internet pour chaque organisme administratif, question d'offrir aux citoyens l'accès à l'information sur les services et les programmes gouvernementaux. Il s'agissait de maximiser le rendement des organismes gouvernementaux et, ultimement, de réduire la taille de la bureaucratie fédérale (Dawes, 2008; Fountain, 2005). Le Presidential Management Agenda lancé par l'administration Bush se concentrait quant à lui sur le développement de projets et d'outils interorganismes pour faciliter l'accès à leurs programmes et services, réduire les coûts de l'accès à l'information gouvernementale, améliorer la communication des renseignements entre les ordres de gouvernement (fédéral, étatique, local et autochtone) et accroître l'efficacité de l'appareil gouvernemental (Fountain, 2005).

L'objectif de l'Open Government Initiative était d'ouvrir l'accès à l'information et au processus décisionnel des organismes fédéraux pour permettre aux citoyens de les examiner et de les commenter et, par le fait même, créer une structure démocratique qui « facilite le jugement de nature sociale et politique que portent les citoyens " (Fung, 2010, p. 107) sur les résultats de l'activité gouvernementale. En outre, l'accessibilité accrue aux renseignements et aux documents gouvernementaux, la possibilité d'apporter de l'information et des points de vue divers au processus décisionnel des organismes gouvernementaux et la capacité des directions d'organisme à s'engager de manière responsable dans ce processus décisionnel constituent des mesures nettement plus démocratiques sur lesquelles repose la vision du gouvernement transparent. Or, à l'échelon fédéral, on assiste au déploiement

3 Pour obtenir un aperçu, consulter la page www.whitehouse.gov/open/documents/flagship-initiatives. 
d'une nouvelle et plus large approche en matière de gouvernance démocratique au moyen des technologies, dont on sait désormais qu'elles peuvent modifier la nature du lien entre les citoyens et les dirigeants de l'appareil administratif.

Pour l'instant, il n'est pas facile de déterminer comment évaluer l'incidence des programmes et des politiques créés précisément pour la réalisation des principes de transparence, de participation et de collaboration. En dépit de l'impression familière et positive qui s'en dégage, il est difficile de juger quelles mesures et quels programmes reflètent ces principes, et selon quel point de vue un tel jugement devrait s'exercer. À titre d'exemple, Sifry (2010, p. 119) rapporte que « ce sont des centaines de milliers de flux de données qui sont mis en ligne à Data.gov, ce qui donne lieu à une toute nouvelle forme d'engagement de la part du public désormais doté d'un accès à l'information ". Même en présumant de l'utilité et de la qualité des données sur lesquelles on peut compter (Allison, 2010; Dawes, 2010), le fait de rendre accessibles de grandes quantités de données sur l'activité gouvernementale constitue-t-il intrinsèquement un acte de "transparence " et quels types de mesures permettent de tirer pareille conclusion? L'apport des citoyens au processus décisionnel d'un organisme gouvernemental fera-t-il en sorte qu'il soit davantage perçu comme " participatif " et comment déterminer le poids des avis des citoyens pour établir une telle conclusion? Ces questions complexes n'ont pas encore fait l'objet d'études rigoureuses.

Dans le présent article, nous abordons la notion de transparence gouvernementale sous l'angle du cybergouvernement ainsi que ses retombées sur l'avenir de l'administration publique, en plus de proposer un cadre conceptuel pour guider les décideurs dans la planification et l'évaluation de leurs programmes de transparence gouvernementale. Nous commençons par associer le gouvernement transparent à deux façons traditionnelles de concevoir la relation entre la technologie, la démocratie et le gouvernement - cyberdémocratie et cybergouvernement - en supposant que l'Open Government Initiative estompe leurs différences par l'intégration de pratiques de tradition démocratique, désormais possibles grâce aux technologies, dans les organismes administratifs. Nous examinons ensuite comment fonctionnent les pratiques démocratiques axées sur la transparence, la participation et la collaboration dans les organes administratifs. Notre analyse suggère que ces valeurs jouent un rôle déterminant dans la création d'un environnement caractérisé par des pratiques démocratiques. Transparence, participation et collaboration pourraient se révéler utiles à l'activité administrative et au processus décisionnel, et non pas en constituer la finalité ni l'objectif. Du moins, elles pourraient contribuer à l'atteinte de fins plus ambitieuses, dont on ne connaît pas encore très bien la nature.

Nous proposons plutôt d'aborder la planification et l'évaluation des programmes et des projets de transparence gouvernementale sous l'angle de la " valeur publique ". Emprunté à des travaux déjà réalisés, ce concept s'appuie sur la nature générale, mais surtout politique, de l'administration publique. La création de valeur publique, à savoir l'information, les programmes et les avantages, constitue l'objectif des organismes publics, car elle leur permet de combler les besoins et les aspirations de la population. Nous appliquons cette vision à la notion de 
transparence gouvernementale pour décrire la valeur dégagée par une interaction gouvernement-citoyens fondée davantage sur la transparence, la participation et la collaboration, c'est-à-dire une interaction plus démocratique. Nous en concluons que les efforts de transparence gouvernementale pourraient ultimement opérer des changements plus profonds dans la structure et l'organisation de la bureaucratie fédérale américaine en faisant connaître les méthodes grâce auxquelles les mécanismes administratifs propices à la transparence, à la participation et à la collaboration produisent des résultats concrets d'une grande valeur au regard des organismes gouvernementaux et des parties prenantes.

\section{- LA TECHNOLOGIE, LA DÉMOCRATIE ET LE GOUVERNEMENT}

L'idée de faire appel aux technologies pour soutenir, améliorer, enrichir, voire revigorer, les pratiques démocratiques n'est pas inédite. L'histoire des médias au XXe siècle a montré que l'apparition de nouveaux moyens de communication donne habituellement lieu à des hypothèses exagérées quant à leurs retombées éventuelles sur les processus et les pratiques démocratiques (Harrison et Falvey, 2001). D'ailleurs, les communications assistées par ordinateur et les technologies de l'information ont fait l'objet d'hypothèses particulièrement excessives qui ont été appliquées aux processus généraux de prise de décision démocratique et de cyberdémocratie ainsi qu'à des formes plus ciblées de mesures gouvernementales comme le cybergouvernement.

Les études sur la cyberdémocratie se concentrent généralement sur la façon dont Internet et les technologies connexes pourraient servir à " hausser la voix politique du citoyen ordinaire " (Hindman, 2009, p. 6) dans les grands processus politiques. Pour y arriver, on élargit l'accès à l'information nécessaire au développement de préférences politiques, on élimine les monopoles bien établis des élites médiatiques sur la diffusion de l'information au profit de nouveaux fournisseurs d'information, on encourage la participation politique lors de campagnes, de référendums et d'élections, on collabore avec les représentants élus et on discute publiquement des politiques.

En revanche, les études sur le cybergouvernement s'intéressent plus directement à l'utilisation que font les organismes publics de la technologie pour accomplir leurs activités ordinaires (Dawes, 2008) : la prestation de services publics, la qualité et le rapport coût-efficacité des activités gouvernementales de base, la mobilisation des citoyens et la consultation de ces derniers, les lois et les mandats législatifs nécessaires à la mise en œuvre de ces processus et les réformes administratives et institutionnelles entreprises pour soutenir les efforts d'innovation. Or, les recherches de Chadwick et May (2003) sur les initiatives de cybergouvernement des administrations américaine, britannique et européenne ont démontré que cellesci avaient privilégié depuis longtemps un mode de "gestion " des interactions entre les citoyens et les organismes fédéraux fondé sur les technologies de l'information et de la communication au détriment d'un mode d'interaction de type participatif et consultatif. Cela ne signifie pas pour autant que la participation et l'engagement sont absents du cybergouvernement. Riley (2003) et Cullen (2010) ont établi les 
différences entre cybergouvernement et cybergouvernance. Cette dernière est définie par les programmes qui invitent les " citoyens à prendre part aux processus politiques de surveillance, en faisant appel à une variété de technologies, notamment le courrier électronique, les réseaux sociaux et les conférences en ligne. La consultation électronique s'appuie sur des systèmes plus formels d'engagement électronique, notamment des initiatives comme le processus électronique d'élaboration de règlements du gouvernement américain (US E-rulemaking) et les projets de cyberparticipation " (Cullen, 2010, p. 58). Cela dit, les administrations présidentielles précédentes, à l'instar des autres ordres de gouvernement, n'ont pas mis la priorité sur les activités de cybergouvernance.

Ce manque d'intérêt peut être attribuable au fait que les organismes administratifs ne sont traditionnellement pas considérés comme des lieux propices à la prise de décisions politiques. En raison du caractère hautement technique qu'on prête aux décisions des administrateurs, car elles servent principalement à mettre en œuvre les mandats législatifs, on estime que les fonctionnaires, qui possèdent vraisemblablement les compétences requises, sont les mieux habilités à accomplir ces tâches. Dans ce contexte, la participation de la population devient inutile. Or, cette approche a récemment fait l'objet de vives critiques. En effet, on remet en question les compétences des administrateurs qui sont nécessaires à la prise de décisions judicieuses (Noveck, 2009). On convient de plus en plus que les organismes administratifs " prennent des décisions qu'ils croient techniques, bien que ce ne soit pas le cas" (Creighton, 2005, p. 14). Les administrateurs choisissent à leur discrétion les modes de conception et de mise en œuvre des politiques; ce faisant, ils posent des jugements de valeur à chaque étape du processus d'élaboration des politiques (Rowe et Frewer, 2000, p. 5). Ces jugements de valeur interviennent dans des questions qui sont l'objet de perceptions contradictoires au sein de la société quant à ce qui est "bon ", ce qui oblige les bureaucrates à concilier l'ensemble des valeurs prônées pour trouver un compromis (Creighton, 2005). Par conséquent, les décisions des organismes administratifs sont loin d'être neutres. Au contraire, elles sont politiques et profondément influencées par les mécanismes de la politique démocratique.

Les organismes administratifs doivent tenir compte de la volonté populaire (Kerwin, 1999), traduite indirectement par l'action des élus. De façon directe, par contre, des lois comme l'Administrative Procedures Act de 1946 ont contraint les administrateurs à consulter la population sur les mesures proposées en matière d'élaboration de réglementation dans différents organismes, tandis que la Federal Advisory Committee Act admet implicitement que l'expertise peut se trouver à l'extérieur de l'appareil administratif, reconnaissant ainsi les bienfaits de la consultation publique. Ces lois n'offrent toutefois que des solutions partielles. L'Open Government Initiative élargit la réceptivité du gouvernement, car elle énonce que les citoyens doivent disposer de l'information pour demander aux organismes fédéraux de rendre compte de leurs actes et intervenir dans leur processus décisionnel.

Ainsi, bien qu'il soit entendu depuis longtemps que la cyberdémocratie intervient dans l'arène politique et que le cybergouvernement entre en scène dans la sphère administrative, il semble que l'actuelle volonté de transparence touche ces 
deux domaines d'activité. Toutefois, même si l'on classe les tentatives d'instauration de la transparence gouvernementale sous la cyberdémocratie ou le cybergouvernement, il n'en demeure pas moins que ces efforts surviennent en l'absence de cadres conceptuels et d'indices de rendement aux fins d'évaluation (voir Lee et Kwak, 2010).

\section{- LA TRANSPARENCE, LA PARTICIPATION ET LA COLLABORATION}

La notion de " gouvernement transparent " est portée par l'optimisme quant à ce qui peut être accompli en politique grâce à l'utilisation des technologies. Elle s'inscrit dans la philosophie et les méthodes du mouvement du logiciel libre ${ }^{4}$. Selon Lathrop et Ruma (2010, p. xix), " à l'instar du logiciel libre dont les utilisateurs peuvent changer et améliorer le code source, le gouvernement transparent signifie que désormais, en plus d'avoir accès à l'information, aux documents et aux procédures, les citoyens peuvent participer de façon significative » à l'action gouvernementale. Les tenants du logiciel libre utilisent les termes transparence, participation et collaboration pour décrire leur mouvement. Toutefois, ceux-ci reflètent aussi des valeurs politiques enracinées depuis fort longtemps dans la théorie démocratique, théorie directement associée au grand processus d'action citoyenne liée au vote et au choix de politiques publiques, mais que l'on souhaite désormais appliquer dans le contexte de l'activité administrative ordinaire au sein de la bureaucratie d'État. Dans les paragraphes qui suivent, nous démontrons comment la transparence, la participation et la collaboration sont devenues prépondérantes dans les contextes administratifs.

\section{La transparence}

Les liens entre information, transparence et démocratie sont fondamentaux. L'information est essentielle à l'établissement des compétences démocratiques de base comme la formulation de préférences et d'opinions, l'évaluation des choix et la participation au processus décisionnel (Doctor, 1992; Van Dijk, 1996). Sans ces compétences, les citoyens ne peuvent se faire entendre sur le marché des idées et se voient privés du droit à la liberté d'opinion et d'expression garantie par le premier amendement (Bowie, 1990). Aussi la "bonne " information, suffisante, rigoureuse et accessible, est-elle une condition préalable à la « bonne » démocratie (Dervin, 1994).

Sans information, les citoyens ne peuvent vraisemblablement pas demander aux gouvernements qu'ils ont élus de rendre compte de leurs actes. Selon De Ferranti (2009, p. 7), le concept de transparence renvoie à « la disponibilité en quantité croissante d'information récente, complète, pertinente, hautement rigoureuse et fiable sur les activités gouvernementales au profit de la population ". Dans une démocratie représentative, où les citoyens délèguent leur autorité décisionnelle, l'accès à l'information est crucial pour jeter les bases d'un consentement

${ }^{4}$ Pour un exposé complet sur le logiciel libre, voir l'article de Guillaume Blum dans ce présent numéro. $[N D L R]$ 
solide. Ainsi le niveau de transparence permet de mesurer le degré de volonté des acteurs gouvernementaux à fournir l'information et les documents dont la population a besoin pour évaluer leurs gestes et exercer son droit de parole dans le cadre du processus décisionnel (Florini, 2007). La Freedom of Information Act habilite les organismes fédéraux à concilier le droit de savoir et les besoins légitimes de protection du secret afin de mettre en place un mécanisme où les citoyens peuvent demander accès à de l'information qui ne peut être rendue publique autrement. Grâce à la divulgation volontaire et régulière des budgets, des vérifications, des politiques et des mesures exécutives, les citoyens peuvent évaluer l'efficacité de l'action administrative et poser des questions sur le type de services publics fournis par le gouvernement; parallèlement, ces gestes stimulent la volonté d'accroître le rendement.

Mais il convient de souligner, comme l'a fait Fung (2010), que la transparence n'est pas le bien absolu. Maximiser les processus de transparence gouvernementale, par exemple, pourrait mettre en évidence une problématique dans le processus de prise de décision, le tout sans le moindre égard aux avantages et aux bénéfices qui en résultent. Fung prétend que les "systèmes de responsabilisation publique " permettraient aux citoyens de fournir une rétroaction continue sur les services gouvernementaux et d'en évaluer les grandes lignes.

En plus de son pouvoir de cultiver l'imputabilité et de contribuer à l'amélioration du rendement du gouvernement, la capacité de la transparence à résoudre les problèmes de légitimité a également fait l'objet de discussions. Comme le soulignent Curtin et Meijer (2006), la transparence peut renforcer la volonté de la population à accepter les structures institutionnelles de plusieurs manières, notamment en faisant clairement état des méthodes employées pour créer les structures d'autorité, en démontrant les avantages tangibles de l'activité institutionnelle et en encourageant les citoyens à croire qu'ils peuvent influencer les décisions institutionnelles et en évaluer les résultats. À l'évidence, il s'agit de questions empiriques. La qualité du lien entre la transparence et ses objectifs hypothétiques n'a toutefois pas encore été pleinement établie (Heald et Hood, 2006).

\section{La participation}

Selon le modèle de la cité athénienne, la démocratie participative, première forme de gouvernance démocratique, n'établit aucune différence entre celui qui gouverne et les citoyens (Held, 1996). Par la discussion directe et, à l'occasion, les délibérations, les citoyens participent directement au processus décisionnel concernant les affaires publiques. De nos jours, hormis au sein des petites collectivités, ces méthodes sont devenues impraticables, ce qui explique l'enthousiasme suscité par l'émergence des nouvelles technologies qui laissent présager la possibilité d'inclure davantage de voix individuelles dans le discours politique et de revitaliser la vie civique et la participation politique (Bertelsen, 1992; Hacker, 1996).

Contrairement au gouvernement représentatif, la démocratie participative s'appuie sur la nécessité pour les citoyens de mieux connaître les points de vue des autres et les intérêts qui les motivent (Rucinski, 1991) en vue de pouvoir en débattre de manière efficace. De nombreux lieux sont le théâtre des échanges d'opinions. Selon Habermas (1991), le lien entre la population et le gouvernement 
démocratique s'enracine dans le discours de l'" espace public ", c'est-à-dire l'interaction sociale entre les citoyens qui discutent des enjeux d'intérêt commun dans divers lieux publics - les cafés, les salons de coiffure, les journaux d'opinion. En démocratie communautaire (Abramson, Arterton et Orren, 1988; Ess, 1996), cette interaction survient dans les lieux de rassemblement neutres ou " great good places " (Oldenburg, 1989) où les citoyens, membres de la communauté, se rencontrent pour discuter des enjeux propices à la vie communautaire et à l'engagement civique. Or, peu importe le lieu, on présume que tous les citoyens disposent du même pouvoir sur la prise de décision et qu'ils l'exercent en toute autonomie.

L'application de ce modèle aux organismes administratifs fait appel à la participation publique pour " intégrer dans le processus décisionnel du gouvernement les préoccupations, les besoins et les valeurs exprimés par la population " (Creighton, 2005, p. 7), qui devient un processus démocratiquement justifié dans la mesure où l'on reconnaît la nature politique des décisions prises par l'administration publique.

La participation publique pourrait contribuer à la diversité des voix citoyennes dans le processus d'élaboration de politiques publiques (OCDE, 2009); l'inclusion des voix traditionnellement exclues pourrait favoriser le positionnement adéquat des politiques dès leur création. On reconnaît généralement que l'équité sociale constitue l'un des principaux objectifs de l'administration publique (Frederickson, 2005) dans l'atteinte duquel la participation publique joue un rôle prépondérant. On peut considérer celle-ci comme un moyen de " redistribuer le pouvoir pour, à l'avenir, sciemment inclure dans les processus politiques et économiques les citoyens dépourvus qui en sont actuellement exclus " (Arnstein, 1969, p. 216) et ainsi prendre des décisions empreintes de justice sociale.

On croit également que la participation publique au processus décisionnel des organismes administratifs pourrait se révéler nettement avantageuse pour les décisions en cause. Les processus de participation publique présentent différentes perspectives dont peuvent s'inspirer les décideurs pour étayer leurs décisions; les citoyens peuvent déterminer aussi bien, voire mieux, que les bureaucrates les solutions qui constituent une politique pertinente, car ils doivent régulièrement faire face aux problèmes (Kweit et Kweit, 1981, p. 72).

Finalement, à l'instar de la transparence, la participation publique peut contribuer de manière significative à résoudre le problème de légitimité des gouvernements actuels (Fung, 2006). Les mesures gouvernementales sont considérées comme légitimes si la population estime avoir de bonnes raisons de les appuyer. Le gouvernement peut accroître la légitimité de ses décisions en tenant compte des intérêts publics dans sa démarche décisionnelle. En démontrant qu'il est sensible aux intérêts exprimés par la population plutôt qu'à ceux des groupes de pression, il obtient l'appui populaire (Fung, 2006).

Bien que la participation publique s'avère particulièrement prometteuse dans le cadre du processus décisionnel de l'administration publique, on reconnaît également qu'elle ne présente pas que des avantages (King, Feltey et Susel, 1998). En effet, la participation accrue n'entraîne pas toujours de meilleurs résultats. Une théorie contingente établit que les différents niveaux de participation sont plus ou 
moins souhaitables selon les caractéristiques et les objectifs du processus d'élaboration des politiques. La portée et la nature de la participation publique devraient dépendre de l'apport éventuel et des possibles conséquences négatives de celle-ci (Cramton, 1971, p. 533). Selon cette approche, la participation comprend trois volets : les intervenants; le mode d'échange d'information et de prise de décisions; le lien entre la participation publique et la prise de décision (Fung, 2006). La place qu'occupent ces trois volets dans la participation publique varie en fonction des résultats désirés.

\section{La collaboration}

Contrairement à la transparence et à la participation, la collaboration n'a pas toujours été directement associée à la théorie politique démocratique. Noveck (2009, p. 19) fait plutôt valoir que la collaboration constitue " une forme de participation démocratique " qui diffère grandement des pratiques participatives et délibérantes conventionnelles qui surviennent souvent dans un contexte nullement lié à la prise de décision. Les débats se transforment souvent en occasions d'échanger des points de vue au lieu de fournir un contexte propice à la détermination d'un plan d'action. Bien que l'inclusion de points de vue variés dans le processus décisionnel gouvernemental amène son lot d'avantages (comme nous l'avons vu plus tôt), Noveck soutient que la collaboration, dans sa forme contemporaine de démocratie participative, réunit des experts et des décideurs pour créer des solutions qui pourront être mises en œuvre.

Cette vision de la collaboration provient de la théorie récente sur l'administration publique synonyme de gestion publique collaborative, à savoir « le processus de facilitation et d'exploitation multiorganisationnelles pour pallier les lacunes insolubles ou difficilement solubles par les organismes individuels " (McGuire, 2006, p. 33), et des modèles analogues, notamment la "nouvelle gouvernance " (Salamon et Elliott, 2002, p. 8). Les gouvernements ont recours à la collaboration pour résoudre les problèmes publics qualifiés de "pernicieux " en raison de la somme des efforts nécessaires à leur résolution. Or, la gestion des " enjeux pernicieux exige un nouveau style de gouvernance. Cette approche s'appuie sur la capacité d'accomplir le travail sans égard aux structures organisationnelles, de penser globalement et de mettre la population à contribution " (Clarke et Stewart, 2003).

Tout comme la participation, la collaboration peut accroître l'efficacité des gouvernements, à condition d'admettre que l'impartialité, l'expertise, les outils, la discipline et le temps nécessaires à la prise de décisions publiques sont des ressources réparties dans la société et de les intégrer au processus d'élaboration de politiques. La collaboration survient quand différents secteurs de la société travaillent de concert, dans un contexte où on reconnaît que les citoyens disposent de renseignements complémentaires potentiellement utiles à la résolution de problèmes publics (Salamon et Elliott, 2002) et que la collaboration peut générer le capital social nécessaire à la participation citoyenne à " valeur ajoutée " (Sirianni, 2009). Le potentiel des méthodes collaboratives s'est accru considérablement avec l'arrivée des nouvelles technologies qui ont donné naissance à un réseau perméable où les frontières organisationnelles n'existent plus (McGuire, 2006). 
Notons cependant que la collaboration fait l'objet de critiques dans les ouvrages traitant d'administration publique. Par exemple, le recours des gouvernements à des acteurs tiers a contribué à cultiver l'image d'État " creux " accolée aux gouvernements qui ont perdu de vue les services qu'ils fournissent à leurs citoyens (Provan et Milward, 2001). Qui plus est, cette pratique soulève des questions de responsabilisation en tenant ces nouveaux participants responsables de leurs actions (Posner, 2002). Les répercussions de la collaboration sur les résultats des programmes sont encore méconnues et, de manière générale, on estime que davantage de collaboration est souhaitable (McGuire, 2006). Ces points de vue reflètent le même rôle d' " intermédiaire " observé pour la transparence et la participation. Comme le souligne Noveck (2009, p. 39), « la collaboration est un moyen de parvenir à une fin. Ainsi, il ne faut pas s'attarder à la participation en soi, mais mobiliser les efforts pour inviter les experts - ceux qui possèdent l'expertise sur un enjeu précis - à participer à la collecte, à l'évaluation et à la pondération des données, ainsi qu'à la création de solutions précises aux fins de mise en œuvre. »

\section{- LE CADRE THÉORIQUE DE LA VALEUR PUBLIQUE}

Les paragraphes précédents montrent clairement que la transparence, la participation et la collaboration sont pour beaucoup dans la concrétisation de la démocratie puisqu'elles permettent aux citoyens de jouer les différents rôles qui leur incombent. Manifestement, la transparence ne constitue pas pour eux une finalité. Les citoyens peuvent souhaiter que leur gouvernement soit transparent, surtout parce qu'il y a autre chose en jeu : l'information et les gestes doivent être transparents pour que les citoyens puissent évaluer et passer au peigne fin les résultats tangibles de l'activité gouvernementale. De la même façon, la participation dans le seul but de participer est un exercice vain et aliénant; il faut plutôt considérer la participation comme un moyen dont disposent les citoyens pour s'assurer que les activités gouvernementales reflètent concrètement et fidèlement leur contribution. La collaboration se révèle pertinente dans la mesure où les citoyens apportent une expertise utile et que des décisions de fond sont envisagées. Bien que ces valeurs ne soient pas une finalité en soi, il est essentiel qu'elles soient clairement reconnues. Les citoyens doivent avoir l'assurance et la certitude que ces valeurs ne serviront pas à accomplir d'autres visées politiques. Parallèlement, comme nous l'avons souligné, plus de transparence, de participation ou de collaboration n'entraîne pas toujours que des avantages. Il faut d'ailleurs faire preuve de prudence pour déterminer les méthodes et les circonstances d'utilisation de ces processus. Ainsi, les mesures qui parviennent à peine à dénombrer les ensembles de données disponibles ou à déterminer la fréquence des occasions propices à la participation ou à la collaboration ne peuvent être vues comme des preuves manifestes de transparence gouvernementale.

Nous soutenons que la transparence, la participation et la collaboration trouvent leur sens lorsqu'elles permettent aux gens de poursuivre leurs objectifs. En supposant que cela soit vrai, quels sont ces objectifs? Dans la section suivante, nous proposons un cadre théorique de la « valeur publique » en vue de déterminer 
la valeur des activités gouvernementales en tenant compte du point de vue des citoyens, certes, mais également de ceux des multiples parties prenantes.

\section{La valeur publique et l'administration publique}

Le concept de la valeur publique, créé par Moore (1995), présume que les organismes publics prennent des décisions qui sont inévitablement de nature politique et que, par conséquent, il appartient aux gestionnaires de déterminer la meilleure façon de les prendre. Sur le modèle des organisations économiques d'appartenance privée qui créent de la "valeur privée " au profit de leurs propriétaires, Moore avance que les organismes publics génèrent de la " valeur publique " au bénéfice des citoyens et d'un large éventail de parties prenantes. La valeur privée provient de l'acquisition de biens et de services à condition qu'elle dégage un profit; il est relativement facile de la repérer et de la mesurer. La valeur publique, pour sa part, est le fruit des avantages engendrés par le gouvernement et survient quand les mécanismes du marché n'arrivent pas à garantir leur production équitable. La valeur publique découle en partie de l'utilité directe de tels avantages, tandis qu'une autre partie provient de l'impartialité et de l'équité de leur production et de leur distribution, sans compter qu'ils doivent satisfaire aux exigences des citoyens en matière d'" institutions publiques hiérarchisées et productives » (Moore, 1995, p. 53).

Cette approche démontre clairement que la rentabilité et l'efficacité ne sont pas nécessairement les seules ni même les principales méthodes d'évaluation des services et des programmes gouvernementaux. Au dire de Moore :

En fin de compte, aucun concept - compétence neutre sur le plan politique, analyse des politiques, évaluation de programmes ou satisfaction de la clientèle - ne peut écarter la politique de son rôle prédominant dans la définition d'une production utile dans le secteur public. La politique demeure l'ultime arbitre de la valeur publique tout comme c'est le cas de la consommation pour la détermination de la valeur privée (Moore, 1995, p. 38).

Chaque citoyen a sa propre opinion sur la valeur relative de l'activité gouvernementale, sauf qu'au final, cette question relève du jugement collectif. L'ampleur de la valeur est susceptible de varier en fonction de l'opinion des groupes d'intérêts et de leur place dans la hiérarchie et peut fluctuer avec le temps. Puisque les avantages de l'action gouvernementale ne sont pas liés au mandat législatif, les gestionnaires publics doivent tenir compte des perceptions de la population à l'égard des programmes et des services offerts par les organismes publics. Moore explique en détail le fonctionnement de ces processus de gestion. Toutefois, il ne propose pas de méthode systématique d'analyse de la valeur publique. Les indices financiers comme la rentabilité, le profit et la productivité ne s'appliquent pas pleinement à ce contexte et c'est pourquoi il faut faire appel à des méthodes comparables pour l'analyse de la valeur publique. Pour pallier cette lacune, Cresswell et ses collègues (Cresswell, Burke et Pardo, 2006; Cresswell, 2010) ont conçu une série de stratégies qui visent à établir un lien entre les intérêts concrets des parties prenantes et des activités gouvernementales précises, en particulier les activités 
relatives aux investissements en technologies de l'information et de la communication destinés à la transparence gouvernementale.

\section{Les retombées de la valeur publique}

En général, la valeur publique met en évidence les intérêts collectifs et sociaux qui sont desservis par diverses institutions et actions gouvernementales. Nous pouvons parler en termes généraux de ces intérêts, mais pour que l'analyse de la valeur publique se révèle profitable, elle doit se concentrer sur les groupes d'intervenants et leurs intérêts particuliers. La répartition de la valeur entre les multiples intervenants varie en fonction de leurs aspirations et de leurs intérêts respectifs à l'égard du gouvernement. Elle varie également selon les avantages que leur procure l'activité gouvernementale, laquelle s'avère parfois nuisible à certaines parties prenantes. Eu égard à cette répartition, si l'on considère la valeur publique comme un indice absolu du rendement de l'activité gouvernementale, on ne tient pas compte de la variété des intérêts ni des avantages possibles pour l'ensemble des parties prenantes. Par le passé, les documents et des déclarations du gouvernement ont associé les initiatives de transparence gouvernementale à la catégorie fourre-tout des " citoyens ». Or, en réunissant une population aussi diversifiée dans un seul groupe et en le traitant comme tel, l'analyse n'arrive malheureusement pas à comprendre la valeur de l'activité gouvernementale. Il s'agit plutôt de conférer à chaque activité gouvernementale une valeur dont pourraient bénéficier les nombreux intervenants, internes et externes. Cette approche nécessite toutefois l'analyse d'une série d'interactions itératives et complexes entre les nombreux intervenants et les activités des organismes et des programmes gouvernementaux.

Le principe fondamental de valeur publique repose sur le lien entre l'action gouvernementale et les retombées diversifiées de la valeur publique qui en découlent. On fait une distinction entre la valeur publique intrinsèque (le bien social) et la valeur substantielle des activités et des politiques gouvernementales qui procurent des avantages précis directs aux divers individus, groupes et organisations. La valeur publique peut entraîner six formes générales de retombées qui couvrent l'ensemble des résultats gouvernementaux possibles selon les intérêts, dont :

- les retombées financières : conséquences actuelles ou futures sur les revenus, la valeur de l'actif, le passif, les droits, les autres formes de richesse ou les risques courus par les éléments précédents;

- les retombées politiques : conséquences sur l'influence des personnes ou des groupes sur l'activité ou les politiques gouvernementales, sur leur rôle dans les affaires politiques, leur pouvoir au sein des partis politiques ou l'attribution des charges publiques;

- les retombées sociales : conséquences sur les familles ou les relations communautaires, la mobilité sociale, le statut socioéconomique et l'identité;

- les retombées stratégiques : conséquences sur les avantages ou les possibilités économiques ou politiques, les objectifs et les ressources nécessaires à l'innovation et à la planification des personnes ou des groupes;

- les retombées idéologiques : conséquences sur les croyances, l'engagement moral ou éthique, l'harmonisation des mesures, des politiques 
gouvernementales ou des résultats sociaux sur les croyances ou les positions morales ou éthiques;

- les retombées administratives : conséquences sur l'image d'honnêtes intendants et gardiens du bien public projetée par les fonctionnaires en ce qui a trait à la confiance publique, à l'intégrité et à la légitimité.

On associe les quatre premières formes de retombées à la valeur substantielle des intérêts privés des individus ou des groupes et les deux autres formes à la valeur intrinsèque, c'est-à-dire aux résultats touchant la société ou la démocratie. La valeur publique du rôle d'intendant endossé par le gouvernement découle de l'intégrité, de la capacité d'adaptation et de la légitimité accrues qui consolident la confiance et la satisfaction à l'égard de l'ensemble des activités gouvernementales. La valeur publique de nature idéologique contribue à l'harmonisation des préférences ou des croyances morales et éthiques.

Une fois ces six formes de retombées de la valeur publique définies, on peut déterminer les enjeux associés aux modes de génération de la valeur. Il suffit de discerner les mécanismes générateurs de valeur, puis de préciser comment l'action gouvernementale est liée à la production d'une ou de plusieurs valeurs publiques. Les mécanismes générateurs de valeur permettent d'établir les cheminements utilitaires qui relient l'action gouvernementale à la valeur qu'elle crée. Notre cadre théorique classe les mesures qui touchent la transparence, la participation et la collaboration parmi les générateurs de valeur suivants :

- la rentabilité : obtenir davantage de revenus ou excéder les objectifs avec les mêmes ressources, ou obtenir les mêmes revenus ou atteindre les objectifs avec moins de ressources;

- l'efficacité : accroître la qualité des résultats anticipés;

- les améliorations intrinsèques : changer l'environnement ou le contexte des parties prenantes à l'aide de moyens dotés d'une valeur propre;

- la transparence : accéder à des renseignements sur les activités des fonctionnaires ou des programmes gouvernementaux qui contribuent à la reddition de comptes ou qui renforcent l'influence du gouvernement;

- la participation : prendre part souvent, pleinement et fréquemment à la prise de décision au sujet des programmes gouvernementaux, aux activités gouvernementales, à la sélection ou aux activités des représentants du gouvernement;

- la collaboration : mobiliser plusieurs parties prenantes pour qu'elles partagent la responsabilité ou exercent une forme d'autorité sur les activités, les politiques ou l'action du gouvernement dans le cadre d'activités de fréquence et de durée variées.

L'association des types de valeurs à leur mécanisme respectif montre clairement comment un programme public peut produire un ou plusieurs types de valeur publique. Par exemple, un investissement en technologies de l'information pour la mise sur pied d'un service en ligne de demandes et de renouvellement de permis peut augmenter la rentabilité et l'efficacité en plus de produire de la valeur publique stratégique ou financière au profit de ceux qui ont besoin de ces permis.

Toute mesure de transparence, de participation et de collaboration entreprise par le gouvernement peut habiliter le citoyen à retirer une valeur substantielle de 
nature financière, sociale, politique ou stratégique ou, encore, une valeur intrinsèque liée au gouvernement. Par exemple, un citoyen disposant de renseignements sur l'environnement (où la transparence joue le rôle de mécanisme générateur de valeur) peut retirer deux types de valeurs. S'il est informé d'un déversement de produits chimiques toxiques dans les environs, il peut faire profiter sa famille et sa collectivité d'avantages de nature sociale en plus de consolider sa confiance dans le rôle d'intendance de l'organisme gouvernemental qui l'a renseigné. En contrepartie, la diffusion d'une telle nouvelle pourrait entraîner des retombées négatives pour certains. À l'annonce d'une pareille nouvelle, le même citoyen pourrait décider de poursuivre en justice le responsable présumé, qui subirait les conséquences de la valeur publique négative dégagée par cette action. Il est aussi possible qu'un certain groupe gouvernemental d'intervenants internes retire une valeur positive de nature politique et stratégique de la diffusion de cette information, puisqu'une telle action correspond aux exigences de transparence gouvernementale, tandis qu'un autre groupe d'intervenants internes pourrait estimer ces retombées négatives sur le plan politique. Par conséquent, déterminer la valeur de l'activité gouvernementale, quelle qu'elle soit, nécessite l'analyse systématique des points de vue des différentes parties prenantes afin de définir et de comprendre les retombées, tant positives que négatives. Les données dégagées de cette analyse rigoureuse serviront à étayer les décisions touchant aux initiatives de transparence gouvernementale.

\section{L'application du cadre théorique de la valeur publique}

Nous travaillons actuellement au perfectionnement de cette approche d'analyse systématique et rigoureuse et devons, à ce stade-ci, prendre en compte certaines incidences. Premièrement, l'analyse de la valeur publique nécessite le recensement relativement exhaustif des parties prenantes de tous les organismes gouvernementaux. Les organes de l'appareil d'État n'interviennent pas directement auprès du " grand public "; leur rôle consiste plutôt à servir des groupes précis, aux exigences et aux besoins particuliers. Aussi doivent-ils pouvoir harmoniser leurs initiatives avec les attentes de leurs parties prenantes.

Deuxièmement, l'analyse de la valeur publique s'appuie sur la capacité des organismes publics de respecter leur mission et leurs priorités dans le cadre des initiatives de transparence gouvernementale. Pour ce faire, ils doivent envisager les éléments fondamentaux de transparence, de participation et de collaboration comme des stratégies dont dispose le gouvernement pour atteindre ses objectifs organisationnels (lesquels devraient être enracinés dans la valeur publique) et produire davantage de valeur par l'intégration de telles pratiques démocratiques. Une information, une participation et une collaboration plus importantes peuvent améliorer le processus décisionnel, puisqu'elles procurent aux parties prenantes des valeurs financières, sociales et stratégiques propices à la hausse de la confiance à l'égard de la capacité de réagir du gouvernement.

Troisièmement, les décideurs du gouvernement peuvent faire appel à cette approche pour planifier, concevoir et évaluer les initiatives de transparence gouvernementale. Pour améliorer les méthodes de conception et de sélection de ces 
initiatives et, ultimement, obtenir les avantages escomptés, il faut déterminer pour chacune : qui en bénéficiera, quelle sera la nature des valeurs créées et quelles mesures généreront ces valeurs. Nous sommes convaincus que cette démarche rationnelle de planification et de conception servira à l'avancement de la planification de la transparence gouvernementale. Les planificateurs peuvent effectuer leur analyse de chaque initiative après avoir ciblé les parties prenantes et les valeurs pour chacune des interventions dont ils ont la responsabilité pour garantir qu'elles s'adressent à un public précis et obtiennent les résultats escomptés. Ils peuvent également analyser l'ensemble des initiatives dont ils ont la responsabilité en déterminant quelles initiatives profitent à chacun des groupes des parties prenantes et de quelles façons elles généreront de la valeur pour garantir que l'organisme remplit son mandat et satisfait aux besoins de ces groupes.

Quatrièmement, les décideurs du gouvernement ont par ailleurs la possibilité d'appliquer cette approche pour évaluer leurs propres initiatives de transparence. Dans ce cas-ci, les parties prenantes, et non la population en général, sont mieux placées pour évaluer une initiative particulière. Qui plus est, au lieu de recourir aux éléments de mesure axés sur les données chiffrées (le nombre de téléchargements, d'occasions de participation ou de billets de discussion, etc.), les organismes doivent évaluer la validité des cheminements utilitaires qui relient une initiative à chacune des parties prenantes ainsi qu'aux valeurs qui s'en dégagent à l'aide des mécanismes générateurs.

Il y a transparence gouvernementale quand les parties prenantes retirent une valeur substantielle ou intrinsèque de l'action gouvernementale accomplie, du moins en partie, dans un esprit de transparence, de participation et de collaboration. Il y a donc lieu de croire que les parties prenantes qui bénéficieront des retombées de telles initiatives percevront le gouvernement comme étant plus sensible, plus accessible, plus engagé et, du coup, plus ouvert.

\section{- CONCLUSION}

Notre vision de la transparence gouvernementale fondée sur la valeur publique et sur les aspirations démocratiques au cœur de cet effort est encore en voie d'élaboration et doit faire l'objet d'une évaluation empirique. En notre qualité de chercheurs du domaine du cybergouvernement, nous croyons que ces travaux sont d'une importance capitale. Notre analyse révèle que nos constructions abstraites du cybergouvernement correspondent grossièrement à celles préconisées par les élus, sans pour autant inspirer les futurs dirigeants. Nous avons intégré les améliorations démocratiques à nos typologies des cybergouvernements, même si elles n'ont pas fait l'objet de travaux dans notre domaine. L'approche du cybergouvernement adoptée par l'administration Obama s'inspire du mouvement du logiciel libre au lieu de s'appuyer sur les recherches en la matière. Dans ce contexte, notre rôle de chercheurs consiste à aider de manière proactive les dirigeants fédéraux à mettre en œuvre, à perfectionner et à évaluer le concept de transparence gouvernementale. 
Cela est d'autant plus important que les concepts sous-jacents de transparence, de participation et de collaboration, éléments eux-mêmes prépondérants, sont souvent mal compris. Les principes de transparence gouvernementale peuvent être assez facilement mis en œuvre. Toutefois, si l'on ne tient pas compte de la valeur publique, cette mise en œuvre pourrait s'avérer futile. Le seul téléchargement d'ensemble de données ne suffit pas à atteindre la transparence. Les ensembles de données doivent s'appuyer sur des renseignements fiables, pertinents et utiles et, par-dessus tout, permettre aux citoyens d'accomplir quelque chose de concret et d'important. Sinon, la transparence n'est qu'une autre promesse en l'air qui accentue le cynisme croissant de l'électorat. De la même manière, la participation et la collaboration doivent être porteuses de sens, orientées vers des objectifs précis; elles doivent faire l'objet de rétroaction abondante de la part du gouvernement. Elles engendrent une contribution des citoyens qui doit faire partie des résultats visibles pour les parties prenantes dans le cadre des décisions et de la valeur produite.

Parallèlement, la transparence gouvernementale concilie les concepts concurrents de cyberdémocratie et de cybergouvernement. Bien que la transparence, la participation et la collaboration nécessitent au départ plus de ressources et de temps, elles permettent tout de même d'espérer un rendement supérieur des politiques publiques - orientation traditionnelle du cybergouvernement - grâce à la compréhension commune du rendement actuel et aux pressions exercées en faveur de l'amélioration, à l'augmentation du réservoir d'idées pertinentes, à l'exploitation de nouvelles sources d'expertise et au renforcement des compétences civiques. Ultimement, tous ces moyens peuvent grandement contribuer à l'amélioration concrète des résultats des politiques publiques et de la qualité des services publics.

L'atteinte de tels résultats repose inévitablement sur les changements de structure et d'organisation au sein du gouvernement. Or Fountain (2005) a constaté que les initiatives de cybergouvernement donnent rarement lieu à des changements structurels concrets. Au contraire, en dépit de leur potentiel novateur, les technologies servent trop souvent à reproduire les règles établies, les habitudes, les normes et les hiérarchies. La promesse de transparence gouvernementale contribue à remédier à ces effets nuisibles. Cette éventualité se réalisera dans la mesure où la transparence gouvernementale parviendra à changer la nature des relations entre les parties prenantes et le gouvernement, créant dans la foulée de nouvelles formes organisationnelles où les groupes interagissent sans égard aux structures organisationnelles ni aux fonctions. La création de valeur publique représente le meilleur argument possible pour stimuler et justifier de tels changements. 


\section{BIBLIOGRAPHIE}

Abramson, J. B., F. C. Arterton et G. R. Orren (1988). The Electronic Commonwealth: The Impact of New Media Technologies on Democratic Politics, Basic Books.

Allison, B. (2010). " My Data Can't Tell You That ", dans D. Lathrop et L. Ruma (dir.), Open Government: Collaboration, Transparency, and Participation in Practice, Sebastopol, O'Reilly Media, Inc., p. 257-265.

Arnstein, S. R. (1969). " A Ladder of Citizen Participation ", Journal of the American Institute of Planners, vol. 35, n 4, p. 216-224.

Barber, B. R. (1984). Strong Democracy: Participatory Politics for a New Age, Berkeley, University of California Press.

Bertelsen, D. A. (1992). "Media Form and Government: Democracy as an Archetypal Image in the Electronic Age ", Communication Quarterly, vol. 40, n 4, p. 325-337.

Bowie, N. (1990). "Equity and Access to Information Technology ", Annual Review of the Institute for Information Studies, p. 131-167.

Chadwick, A. et C. May (2003). "Interaction between States and Citizens in the Age of the Internet: "e-Government" in the United States, Britain, and the European Union ", Governance an International Journal of Policy and Administration, vol. 16, n ${ }^{\circ} 2$, p. 271-300.

Clarke, M. et J. Stewart (2003). "Handling the Wicked Issues ", dans J. Reynolds et autres (dir.), The Managing Care Reader, London, Routledge, p. 273-280.

Cramton, R. C. (1971). "The Why, Where and How of Broadened Public Participation in the Administrative Process ", The Georgetown Law Journal, vol. 60, n 3, p. 1-26.

Creighton, J. L. (2005). The Public Participation Handbook: Making Better Decisions through Citizen Involvement, Jossey-Bass Inc Pub.

Cresswell, A. M., G. B. Burke et T. Pardo (2006). Advancing Return on Investment, Analysis for Government IT: A Public Value Framework, Albany, Center for Technology in Government.

Cresswell, A. M. (2010). Public Value and Government ICT Investment, Antalya, Turkey.

Cullen, R. (2010). " Defining the Transformation of Government: E-Government or e-Governance Paradigm ", dans H. J. Scholl (dir.), E-Government: Information, Technology, and Transformation, M.E. Sharpe, p. 57-71.

Curtin, D. et A. J. Meijer (2006). " Does Transparency Strengthen Legitimacy? ", Information Polity, vol. 11, $\mathrm{n}^{\circ}$ 2, p. 109-122.

Dawes, S. S. (2010). "Stewardship and Usefulness: Policy Principles for Informationbased Transparency ", Government Information Quarterly, vol. 27, n 4, p. 377-383.

Dawes, S. S. (2008). « The Evolution and Continuing Challenges of e-Governance ", Public Administration Review, vol. 68, p. S86-S102.

Dervin, B. (1994). "Information $\leftrightarrow$ Democracy: An Examination of Underlying Assumptions ", Journal of the American Society for Information Science, vol. 45, $\mathrm{n}^{\circ} 6$, p. 369-385.

Doctor, R. D. (1992). " Social Equity and Information Technologies: Moving toward Information Democracy ", Annual Review of Information Science and Technology, vol. 27, p. 43-96. 
Ess, C. (1996). « The Political Computer: Democracy, CMC, and Habermas », dans C. Ess (dir.), Philosophical Perspectives on ComputerMediated Communication, Albany, State University of New York Press, p. 197-230.

Ferranti, D. (de) (2009). How to Improve Governance: A New Framework for Analysis and Action, Brookings Institution Press.

Florini, A. (2007). The Right to Know: Transparency for an Open World, Columbia University Press.

Fountain, J. (2005). " Central Issues in the Political Development of the Virtual State ", dans M. Castells et G. Cardoso (dir.), The Network Society: From Knowledge to Policy, Washington D.C., Center for Transatlantic Relations, p. 149-181.

Frederickson, H. G. (2005). " Public Administration and Social Equity », dans J. Beckett et H. O. Koenig (dir.), Public Administration and Law, ASPA, p. 209-222.

Fung, A. (2010). "Open Government and Open Society ", dans D. Lathrop et L. Ruma (dir.), Open Government: Collaboration, Transparency, and Participation in Practice, Sebastopol, O’Reilly Media, Inc., p. 105-113.

Fung, A. (2006). "Varieties of Participation in Complex Governance ", Public Administration Review, numéro spécial, vol. 66, p. 66-75.

Habermas, J. (1991). The Structural Transformation of the Public Sphere: An Inquiry into a Category of Bourgeois Society, MIT Press.

Hacker, K. L. (1996). "Missing Links in the Evolution of Electronic Democratization ", Media, Culture and Society, vol. 18, n² 2, p. 213-232.

Harrison, T. et L. Falvey (2001). « Democracy and New Communication Technologies ", dans W. B. Gudykunst (dir.), Communication yearbook 25, Lawrence Erlbaum Associates, p. 1-43.

Heald, D. et C. Hood (2006). Transparency: The Key to Better Governance?, Oxford University Press.

Held, D. (1996). Models of Democracy, Stanford University Press.

Hindman, M. S. (2009). The Myth of Digital Democracy, Princeton University Press.

Kerwin, C. M. (1999). Rulemaking: How Government Agencies Write Law and Make Policy, CQ Press College.

King, C. S., K. M. Feltey et B. O. Susel (1998). "The Question of Participation: Toward Authentic Public Participation in Public Administration ", Public Administration Review, vol. 58, n 4, p. 317-326.

Kweit, M. G. et R. W. Kweit (1981). Implementing Citizen Participation in a Bureaucratic Society: A Contingency Approach, New York, Praeger.

Lathrop, D. et L. Ruma (2010). Open Government: Collaboration, Transparency, and Participation in Practice, Sebastopol, O'Reilly Media, Inc.

Lee, G. et Y. Kwak (2010). Open Government Implementation Model: Moving to Increased Public Engagement, www.businessofgovernment.org/report/open-governmentimplementation-model-moving-increased-public-engagement (page consultée le 2 octobre 2011).

McGuire, M. (2006). « Collaborative Public Management: Assessing What We Know and How We Know It ", Public Administration Review, vol. 66, Issue Supplement s1, p. 33-43. 
Moore, M. H. (1995). Creating Public Value: Strategic Management in Government, Harvard University Press.

Noveck, B. S. (2009). Wiki Government: How Technology Can Make Government Better, Democracy Stronger, and Citizens More Powerful, Brookings Inst Pr.

Obama, B. (2009). Transparency and Open Government: Memorandum for the Heads of Executive Departments and Agencies, www.whitehouse.gov/the_press_office/ TransparencyandOpenGovernment (page consultée le 2 octobre 2011).

OCDE (2009). Focus on Citizens: Public Engagement for Better Policy and Services, OCDE.

Oldenburg, R. (1989). The Great Good Place: Cafes, Coffee Shops, Community Centers, Beauty Parlors, General Stores, Bars, Hangouts, and How They Get You Through the Day, Paragon House.

Orszag, P. (2009). Open Government Directive, www.whitehouse.gov/open/documents/ opengovernment-directive (page consultée le 2 octobre 2011).

Posner, P. L. (2002). "Accountability Challenges of Third Party Governance ", The Tools of Government, New York, Oxford University Press.

Provan, K. G. et H. B. Milward (2001). « Do Networks Really Work? A Framework for Evaluating Public-Sector Organizational Networks ", Public Administration Review, vol. $61, n^{\circ} 4$, p. 414-423.

Riley, T. (2003). E-Governance us. e-Government, www.i4donline.net/articles/currentarticle. asp ?articleid=453\&typ=Features (page consultée le 2 octobre 2011).

Rowe, G. et L. J. Frewer (2000). " Public Participation Methods: A Framework for Evaluation ", Science, Technology \& Human Values, vol. 25, n 1, p. 329.

Rucinski, D. (1991). " The Centrality of Reciprocity to Communication and Democracy ", Critical Studies in Mass Communication, vol. 8, n 2, p. 184-194.

Salamon, L. M. et O. V. Elliott (2002). The Tools of Government: A Guide to the New Governance, Oxford University Press.

Sifry, M. (2010). "You Can Be the Eyes and Ears: Barack Obama and the Wisdom of Crowds ", dans D. Lathrop et L. Ruma (dir.), Open Government: Collaboration, Transparency, and Participation in Practice, Sebestopol, O'Reilly Media, Inc., p. 115-122.

Sirianni, C. (2009). Investing in Democracy: Engaging Citizens in Collaborative Governance, Brookings Institution Press.

Stirland, S. (2008). Obama's Secret Weapons: Internet, Databases and Psychology, http://www. wired.com/threatlevel/2008/10/obamas-secretw/ (page consultée le 2 octobre 2011).

Stone, D. et D. Lyons (2008). President 2.0, http://www.newsweek.com/2008/11/21/ president-2- 0.print.html (page consultée le 2 octobre 2011).

Van Dijk, J. A. (1996). « Models of Democracy: Behind the Design and Use of New Media in Politics ", Electronic Journal of Communication, vol. 6, n 2.

Wagner, M. (2008). Obama Election Ushering in First Internet Presidency, www.informationweek. com/news/government/showArticle.jhtml?articleID=212000815 (page consultée le 2 octobre 2011). 